\title{
Artemisinin Improves Acetylcholine-Induced Vasodilatation in Rats with Primary Hypertension
}

\author{
Xuanxuan Liu, ${ }^{1,2}$ Xingxing \\ Wang,' Yan Pan,' Li Zhao, ${ }^{3}$ \\ Shuo Sun,' Ang Luo, ${ }^{3}$ \\ Changlei Bao, ${ }^{3,4}$ Haiyang \\ Tang, (D) ${ }^{3,4}$ Ying Han (D) ${ }^{1}$
}

'Key Laboratory of Targeted Intervention for Cardiovascular Disease, Collaborative Innovation Center of Translational Medicine for Cardiovascular Disease, Department of Physiology, Nanjing Medical University, Nanjing, Jiangsu, People's Republic of China; ${ }^{2}$ Department of Physiology and Pathologic Physiology, Kangda College of Nanjing Medical University, Lianyungang, Jiangsu, People's Republic of China; ${ }^{3}$ College of Veterinary Medicine, Northwest A\&F University, Yangling, Shaanxi, People's Republic of China; ${ }^{4}$ State Key Laboratory of Respiratory Disease, National Clinical Research Center for Respiratory Disease, Guangzhou Institute of Respiratory Health, The First Affiliated Hospital of Guangzhou Medical University, Guangzhou, Guangdong, People's Republic of China

Correspondence: Ying Han Key Laboratory of Targeted Intervention of Cardiovascular Disease, Collaborative Innovation Center of Translational Medicine for Cardiovascular Disease, Department of Physiology, Nanjing Medical University, Nanjing, Jiangsu, 21 II66, People's Republic of China Tel/Fax +86-25-8686935 I

Email yhancn@njmu.edu.cn

Haiyang Tang

State Key Laboratory of Respiratory

Disease, Guangzhou Institute of Respiratory Health, The First Affiliated

Hospital of Guangzhou Medical

University, Guangzhou, Guangdong,

5 I0120, People's Republic of China

Tel +86-15975396262

Email tanghy2008@yahoo.com
Purpose: Endothelial dysfunction and the subsequent decrease in endothelium-dependent vascular relaxation of small arteries are major features of hypertension. Artemisinin, a wellknown antimalarial drug, has been shown to exert protecting roles against endothelial cell injury in cardiac and pulmonary vascular diseases. The current study aimed to investigate the effects of artemisinin on endothelium-dependent vascular relaxation and arterial blood pressure, as well as the potential signalling pathways in spontaneously hypertensive rats (SHRs).

Methods: In this study, acetylcholine ( $\mathrm{ACh}$ )-induced dose-dependent relaxation assays were performed to evaluate vascular endothelial function after treatment with artemisinin. Artemisinin was administered to the rats by intravenous injection or to arteries by incubation for the acute exposure experiments, and it was administered to rats by intraperitoneal injection for 28 days for the chronic experiments.

Results: Both acute and chronic administration of artemisinin decreased the heart rate and improved ACh-induced endothelium-dependent relaxation but negligibly affected the arterial blood pressure in SHRs. Incubation with artemisinin decreased basal vascular tension, $\mathrm{NAD}(\mathrm{P}) \mathrm{H}$ oxidase activity and reactive oxygen species (ROS) levels, but it also increased endothelial nitric oxide (NO) synthase (eNOS) activity and NO levels in the mesenteric artery, coronary artery, and pulmonary artery of SHRs. Artemisinin chronic administration to SHRs increased the protein expression of eNOS and decreased the protein expression of the $\mathrm{NAD}(\mathrm{P}) \mathrm{H}$ oxidase subunits NOX-2 and NOX-4 in the mesenteric artery.

Conclusion: These results indicate that treatment with artemisinin has beneficial effects on reducing the heart rate and basal vascular tension and improving endothelium-dependent vascular relaxation in hypertension, which might occur by increasing eNOS activation and NO release and inhibiting NAD(P)H oxidase derived ROS production.

Keywords: hypertension, artemisinin, vascular function, nitric oxide, reactive oxygen species

\section{Introduction}

Endothelial cells play a critical role in maintaining vascular homeostasis. ${ }^{1}$ Endothelial dysfunction and the consequent imbalance between vasodilation and vasoconstriction function are the major pathophysiological factors contributing to cardiovascular diseases, including hypertension. ${ }^{2}$ Increased vascular constriction and attenuated endothelium-dependent vasorelaxation due to endothelial dysfunction of small arteries are important hallmarks of hypertension. ${ }^{3}$ As acetylcholine (ACh) primarily stimulates endothelial cells to release nitric oxide (NO) ${ }^{4}$ and induce vascular smooth muscle cell relaxation, it has been widely used to evaluate vascular endothelial function. ${ }^{5}$ Sodium nitroprusside, an NO donor that directly 


\section{Graphical Abstract}

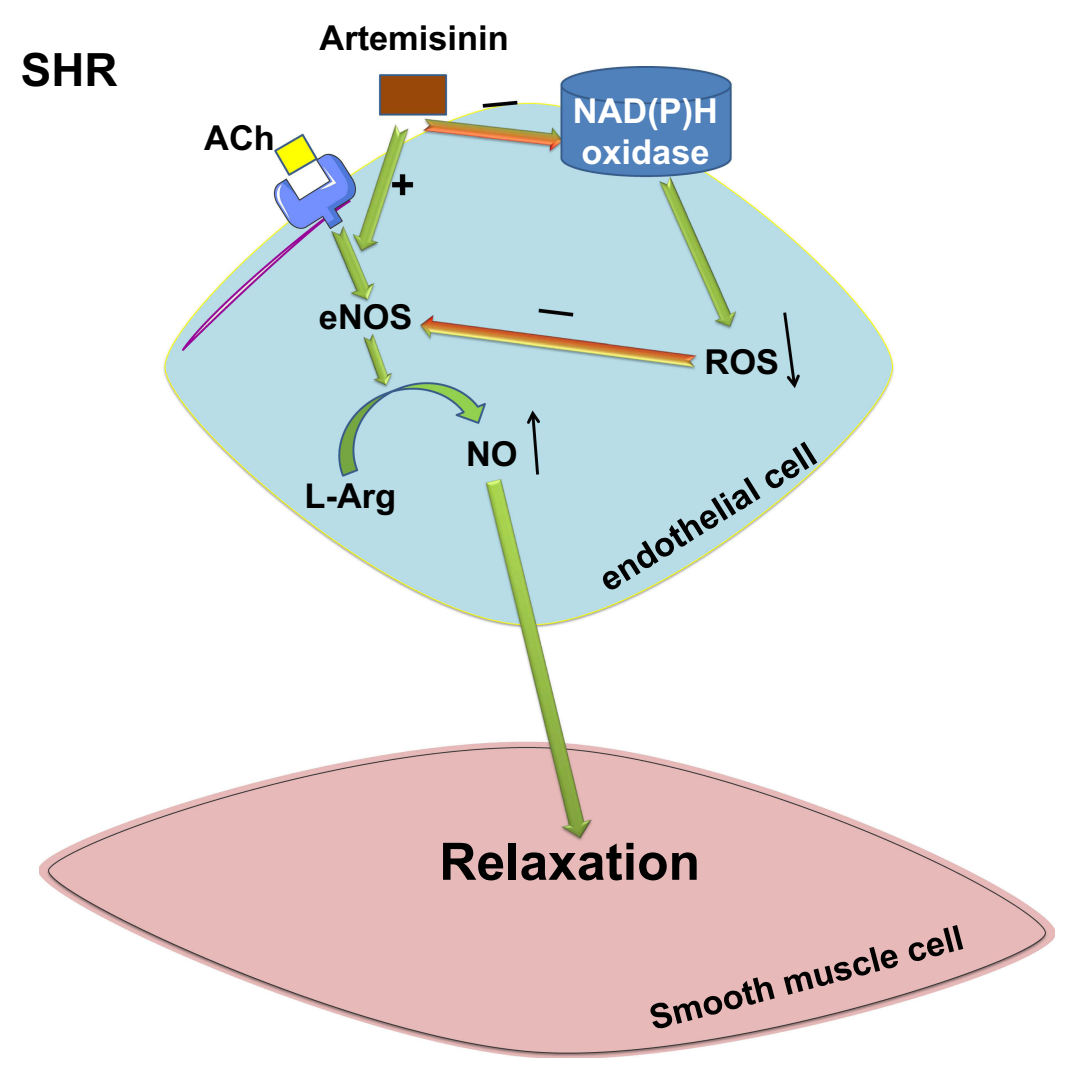

induces vascular smooth muscle cell relaxation, is commonly used to evaluate endothelium-independent relaxation. ${ }^{6}$ In our previous studies, we demonstrated that ACh-induced vasodilatation of three important small arteries, the mesenteric artery, pulmonary artery and coronary artery, of spontaneously hypertensive rats (SHRs) was significantly attenuated. ${ }^{7,8}$ Subsequently, elevated peripheral and pulmonary vascular resistance increases the risk of insufficient blood supply to the myocardium and the risk of systemic and pulmonary hypertension. However, the mechanisms involved in the impaired vascular relaxation function of small arteries in hypertension remain unclear.

Artemisinin is extracted from the traditional Chinese herb Artemisia annua and it is widely used as a first-line antimalarial drug with relatively low toxicity. Recently, artemisinin and its derivatives, including dihydroartemisinin and artesunate, have been found to play beneficial roles in a number of other maladies, including cardiovascular diseases., ${ }^{9,10}$ They have been reported to reduce atherosclerotic lesions, ${ }^{11,12}$ alleviate rat cardiac hypertrophy, ${ }^{13}$ attenuate pulmonary hypertension by inhibiting pulmonary vascular remodelling in rats, ${ }^{14,15}$ and inhibit obesity, diabetes and fatty liver. ${ }^{16,17}$ Their beneficial roles include anti-inflammatory, ${ }^{18,19}$ anti-fibrosis, ${ }^{14}$ antiproliferation and anti-migration, ${ }^{18,20}$ antitumour ${ }^{21}$ and antiviral activity, $^{22}$ and anti-neovascularization effects in diseases dependent on new blood vessel formation ${ }^{20,23,24}$ in addition to their antimalarial activity.

More importantly, they have protective roles on endothelial cells. Dihydroartemisinin effectively inhibits hypoxia-induced cell proliferation, migration, and oxidative stress in human pulmonary artery endothelial cells. ${ }^{15}$ Artesunate has demonstrated a protective effect against lipopolysaccharide-induced injury of human umbilical vein endothelial cells. ${ }^{19}$ Treatment with artesunate remarkably inhibits the proliferation and differentiation of endothelial cells in a dose-dependent manner. ${ }^{23}$ More recently, studies have reported that treatment of haemorrhagic shock rats with artesunate enhances the activation of endothelial nitric oxide synthase (eNOS). ${ }^{25}$ In alveolar 
macrophages, artesunate alleviates hypoxia/reoxygenation-induced increases in reactive oxygen species (ROS) production. $^{26}$ Furthermore, we recently found that decreased eNOS-NO release and increased ROS production in endothelial cells are important factors in attenuating endothelium-dependent vascular relaxation in primary hypertension. ${ }^{27,28}$ However, the effect of artemisinin on endothelial dysfunction in hypertension remains unknown.

The present study was therefore designed to determine whether artemisinin improves endothelial function and redresses attenuated vascular relaxation function in three arteries, the mesenteric artery (MA), pulmonary artery (PA) and coronary artery (CA), of SHRs and whether it affects blood pressure in primary hypertension. In addition, the potential molecular mechanisms underlying the roles of artemisinin in SHRs were also explored.

\section{Materials and Methods}

Thirteen-week-old male SHRs and Wistar-Kyoto rats (WKYs) (Vital River Laboratory Animal Technology Co. Ltd., Beijing, China), which were the controls for the SHRs, were used in this experiment. The rats were housed in a humidity- and temperature-controlled animal room with free access to standard chow and tap water on a 12 h-12 h light-dark cycle. All procedures were approved by the Nanjing Medical University Experimental Animal Care committee and complied with the Guide for the Care and Use of Laboratory Animals published by the US National Institutes of Health (NIH publication, 8th edition, 2011). The major methods used in this study were performed as described in a previous report. ${ }^{28}$

\section{Systolic Blood Pressure Measurement}

A noninvasive computerized tail-cuff system (Kent Scientific Corporation, CT, USA) was used to measure the systolic blood pressure of the tail artery in conscious rats. The systolic blood pressure was obtained by averaging 10 measurements as described in our previous reports. ${ }^{28,29}$

\section{Heart Rate and Mean Arterial Pressure Recording}

Rats were anaesthetized by intraperitoneal injection with urethane $(800 \mathrm{mg} / \mathrm{kg})$, and then the right carotid artery was cannulated. A pressure transducer (MLT0380, ADInstruments, Australia) linked to a Powerlab data acquisition system (8SP, ADInstruments, Australia) was used to continuously record the arterial blood pressure, from which the heart rate and mean arterial pressure under anaesthesia were calculated using Chart software.

\section{Intravenous Injection}

Artemisinin was administered to the rats by intravenous injection to record the acute influence of artemisinin on blood pressure. External jugular vein catheterization was performed for intravenous injection of saline, DMSO, and artemisinin $(0.33 \mathrm{mg} / \mathrm{kg})$, and then the effects of these chemicals on mean arterial pressure and heart rate in WKYs and SHRs were analysed over one hour. A dual-channel microdialysis infusion syringe pump (53101 V, Stoelting Co., Illinois, USA) was used to control the injection rate. For each injection, $100 \mu \mathrm{L}$ was injected over 10 minutes.

\section{Measurement of Isometric Arterial Tension}

An isometric tension experiment was performed to evaluate the vascular relaxation function as described in our previous reports. ${ }^{7,30}$ Briefly, we first isolated third-order $\mathrm{MA}, \mathrm{CA}$ and PA from rats and cut them into 1 to $1.2 \mathrm{~mm}$ segments under a microscope in Krebs-Henseleit solution (the constituents of which can be found in our previous study ${ }^{7,30}$ ) in a dish placed with a scale on the bottom by using a special pair of scissors with a fine point (World Precision Instruments Inc. USA). Then, we mounted the arterial rings ( 1 arterial ring/artery/rat was used) in a fourchambered myograph (620M, DMT, Denmark) and subjected them to resting tension at $0.1 \mathrm{~g}$.

High $\mathrm{K}^{+}$solution (its constituents can be found in our previous studies, ${ }^{7,30}$ and it includes $122.6 \mathrm{KCl}, 1.09 \mathrm{CaCl}_{2}$ $\cdot 7 \mathrm{H}_{2} \mathrm{O}, 1.21 \mathrm{MgSO}_{4} \cdot 7 \mathrm{H}_{2} \mathrm{O}, 1.117 \mathrm{KH}_{2} \mathrm{PO}_{4}, 24.9 \mathrm{KHCO}_{3}$ and 11.1 glucose (in $\mathrm{mmol} / \mathrm{L}$ )) was used to evaluate the vasoconstriction function. ACh (6 doses: $10^{-9} \sim 10^{-4} \mathrm{~mol} /$ L) or sodium nitroprusside (6 doses: $10^{-9} \sim 10^{-4} \mathrm{~mol} / \mathrm{L}$ ) were administered after arterial ring contraction induced by prostaglandin F $2 \alpha$ (PGF $2 \alpha)(1-5 \mu \mathrm{mol} / \mathrm{L})$ to evaluate the endothelium-dependent vascular relaxation or endothelium-independent relaxation. The degree of vascular relaxation is shown as a percentage of PGF $2 \alpha$-induced contraction. Pretreatment with saline, $0.1 \%$ DMSO or three doses of artemisinin $(2,20,200 \mu \mathrm{mol} / \mathrm{L})$ was performed one hour before PGF $2 \alpha$ or high $\mathrm{K}^{+}$solutioninduced contraction. The basal vascular tension before and after drug application to the arteries were measured using Chart software of the Powerlab system. The change 
in basal vascular tension induced by drug incubation was then calculated by the difference in the value before and after drug incubation.

The dose and concentration of artemisinin were defined according to a previous report ${ }^{31}$ and the results of our preliminary experiments. All of the concentrations referred to in this study were the final concentrations in the KrebsHenseleit solution in the chamber of the myograph.

\section{Chronic Application of Artemisinin}

Intraperitoneal injection of artemisinin every day for 28 days was performed to determine its effect on blood pressure and other functions after long-term application. Artemisinin was administered to the rats by intraperitoneal injection at $60 \mathrm{mg} / \mathrm{kg} / \mathrm{day}$ for 28 days following the methods used in a previous report, ${ }^{32}$ and equivalent amounts of DMSO were injected as a vehicle control. On the $29^{\text {th }}$ day, the systolic blood pressure in the conscious state and the mean arterial pressure and heart rate under anaesthesia were measured. Then, isometric tension measurements of MA, CA and PA were performed to test ACh-induced endothelium-dependent relaxation or sodium nitroprusside-induced endothelium-independent relaxation.

\section{Measurement of Arterial eNOS Activity and NO Levels}

Third-order MA, CA and PA samples with the same length $(5 \mathrm{~mm})$ were isolated from the rats and incubated separately in Krebs-Henseleit solution containing saline, 0.1\% DMSO and artemisinin $(20 \mu \mathrm{mol} / \mathrm{L})$ for 1 hour. At the same time, a nitric oxide synthase assay kit (Beyotime Biotech Inc., Nanjing, China) was used to evaluate eNOS activity in the arteries by testing the conversion of L-arginine to NO following the manufacturer's instructions.

For measurement of the NO levels, the artery tissues were homogenized in chilled RIPA buffer and centrifuged at $4{ }^{\circ} \mathrm{C}$ after incubation, and then the supernatant was extracted and measured using a BCA assay (BCA, Pierce, USA). NO production in the arteries was assessed using a nitrate/nitrite colorimetric assay kit (Cayman Chemical Co., Ann Arbor, MI, USA) following the manufacturer's instructions.

\section{Measurement of $\mathrm{NAD}(\mathrm{P}) \mathrm{H}$ Oxidase Activity and Superoxide Anion Levels in the Arteries}

$\mathrm{NAD}(\mathrm{P}) \mathrm{H}$ oxidase activity and superoxide anion levels of the arteries after incubation with different chemicals were measured using an enhanced lucigenin-derived chemiluminescence method as previously reported. ${ }^{28,33}$ Briefly, the light emissions produced from the reaction between lucigenin $(5 \mu \mathrm{M})$ and the superoxide anions in the artery tissue homogenate supernatant were recorded by a luminometer (20/20n, Turner, CA, USA) once every minute for 10 minutes to measure the superoxide anion levels. For the $\mathrm{NAD}(\mathrm{P}) \mathrm{H}$ oxidase activity measurement, the $\mathrm{NAD}(\mathrm{P}) \mathrm{H}$ substrate $(100 \mu \mathrm{M})$ was added to the medium to react with $\mathrm{NAD}(\mathrm{P}) \mathrm{H}$ oxidase to generate superoxide anions before the light emissions were detected by the luminometer.

\section{Protein Expression in the Arteries Measured by Western Blotting}

After the third-order MA samples were isolated from the rats and frozen, they were homogenized and centrifuged. The protein concentration was measured using a protein assay kit (BCA, Pierce, USA). The protein was loaded onto an SDS-PAGE gel and then transferred to a polyvinylidene fluoride membrane. The membranes were then probed overnight at $4{ }^{\circ} \mathrm{C}$ with antibodies against eNOS (1:1000, Cell Signaling Technology, USA), NOX-2 (1:1000, Proteintech Inc., Wuhan, China), NOX-4 (1:1000, Proteintech Inc., Wuhan, China), or $\beta$-actin (1:5000, Abways Technology Inc., Shanghai, China) followed by incubation with horseradish peroxidaseconjugated goat anti-rabbit IgG (1:5000, Immunology Consultants Lab, Portland, OR, USA). Protein loading was controlled by probing all blots with a $\beta$-actin antibody. The bands were visualized by an enhanced chemiluminescence ECL system (Pierce Chemical, Rockford, IL, USA).

\section{Chemicals}

Prostaglandin F2 $\alpha$ (PGF 2 $\alpha$ ), acetylcholine (ACh), and NAD $(\mathrm{P}) \mathrm{H}$ were ordered from Sigma (St. Louis, MO, USA). Artemisinin was purchased from the National Institutes for Food and Drug Control of China. Most of the chemicals were dissolved in normal saline, except for artemisinin, which was dissolved in dimethyl sulfoxide (DMSO).

\section{Statistical Analysis}

Emax and pD2 data were calculated using GraphPad Prism software. SPSS analysis software was used to perform the statistical analyses. All values are presented as the mean $\pm \mathrm{S}$. 
E. Student's unpaired $t$-Test was used for comparisons between two groups. One-way or two-way ANOVA followed by Bonferroni's post hoc analysis was used for multiple comparisons. $P<0.05$ was considered statistically significant.

\section{Results}

\section{Effects of Intravenous Injection of Artemisinin on the Mean Arterial Pressure and Heart Rate in WKYs and SHRs}

To investigate whether artemisinin directly influences arterial blood pressure, we examined the effects of intravenous injection of artemisinin on arterial blood pressure in WKYs and SHRs in vivo. We found that both the systolic blood pressure and mean arterial pressure in the SHRs were higher than those of the WKYs. There was no significant difference in the body weight or heart rate between the WKYs and SHRs (Table 1). There was also no significant difference between the effects of DMSO and saline on the rats. Intravenous injection of artemisinin had no significant effect on the mean arterial pressure or heart rate of the WKYs. Compared to DMSO or saline, intravenous injection of artemisinin decreased the heart rate but had no significant effect on the mean arterial pressure of the SHRs (Figure 1).

\section{Effects of Artemisinin on the Basal Vascular Tension in WKYs and SHRs}

To determine whether artemisinin directly induces vasodilation or vasoconstriction, we examined the effects of MA, $\mathrm{CA}$ and PA treatment with three doses of artemisinin (2, 20 , and $200 \mu \mathrm{mol} / \mathrm{L}$ ) for an hour on the basal vascular tension in WKYs and SHRs. Treatment of MA, CA and
Table I The SBP, MAP, HR and Body Weight in One Representative Group of WKYs and SHRs

\begin{tabular}{|l|l|l|}
\hline & WKY & SHR \\
\hline SBP, mm Hg & $118.7 \pm 6.4$ & $187.6 \pm 5.7^{*}$ \\
MAP, mm Hg & $90.6 \pm 4.7$ & $133.8 \pm 4.0^{*}$ \\
HR, beats/min & $350.2 \pm 13.1$ & $353.4 \pm 12.3$ \\
Body Weight, g & $320.5 \pm 4.8$ & $327.1 \pm 5.3$ \\
\hline
\end{tabular}

Notes: The SBP (systolic blood pressure) of tail artery of rats in conscious state was measured using a noninvasive computerized tail-cuff system. The MAP (mean arterial pressure) and HR (heart rate) were measured by a pressure transducer in the right carotid artery under anesthesia. Values are expressed as mean $\pm \mathrm{SE}$. $* \mathrm{P}<0.05$ compared with the WKY rats. $\mathrm{n}=6$ for each group.

PA with all three doses of artemisinin did not significantly affect the basal vascular tension in the WKYs. In the SHRs, the basal vascular tension of MA was decreased in response to all three doses of artemisinin, while the middle dose of artemisinin had the strongest effect. For CA from SHRs, the low and middle doses of artemisinin decreased the basal vascular tension, and the low dose had the strongest effect. The PA from SHRs behaved similarly to that of CA from SHRs. These effects of artemisinin were not dose-dependent in SHRs (Figure 2).

\section{Effects of Artemisinin on Endothelium-Dependent Vascular Relaxation in WKYs and SHRs}

To investigate the influence of artemisinin on endothelial function, we examined the effects of pretreatment with three doses of artemisinin $(2,20,200 \mu \mathrm{mol} / \mathrm{L})$ on AChinduced dose-dependent vascular relaxation of MA, CA and PA from WKYs and SHRs. Pretreatment with the three doses of artemisinin had no significant effect on ACh-induced vascular relaxation of MA, CA or PA from

B

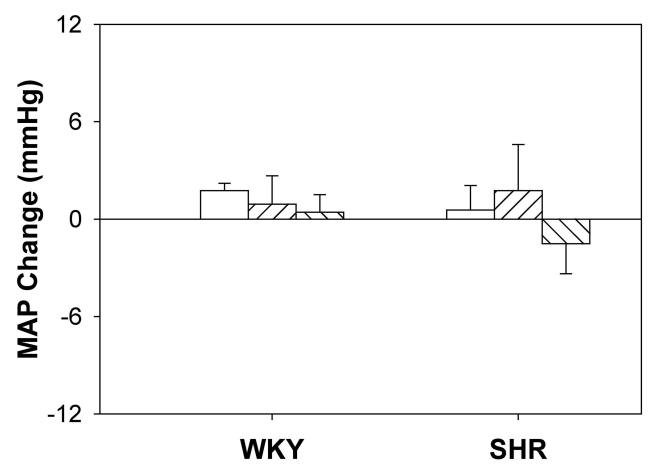

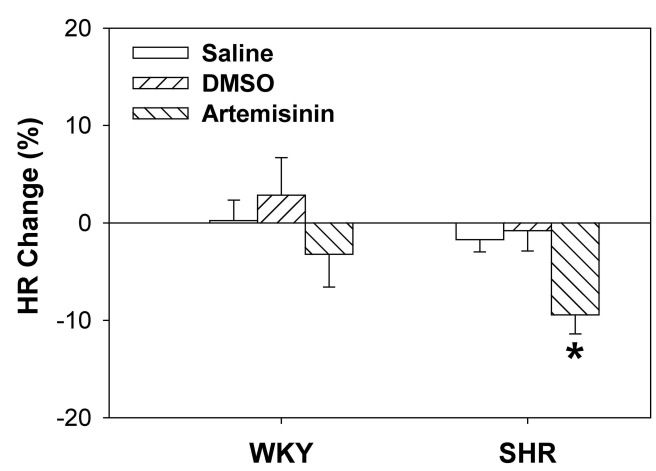

Figure I Effects of acute intravenous injection of saline, DMSO, and artemisinin $(0.33 \mathrm{mg} / \mathrm{kg})$ on the mean arterial pressure (A) and heart rate (B) of WKYs and SHRs. Data represent mean \pm SE. $* P<0.05$ compared to saline or DMSO; $n=6$ for each group. 

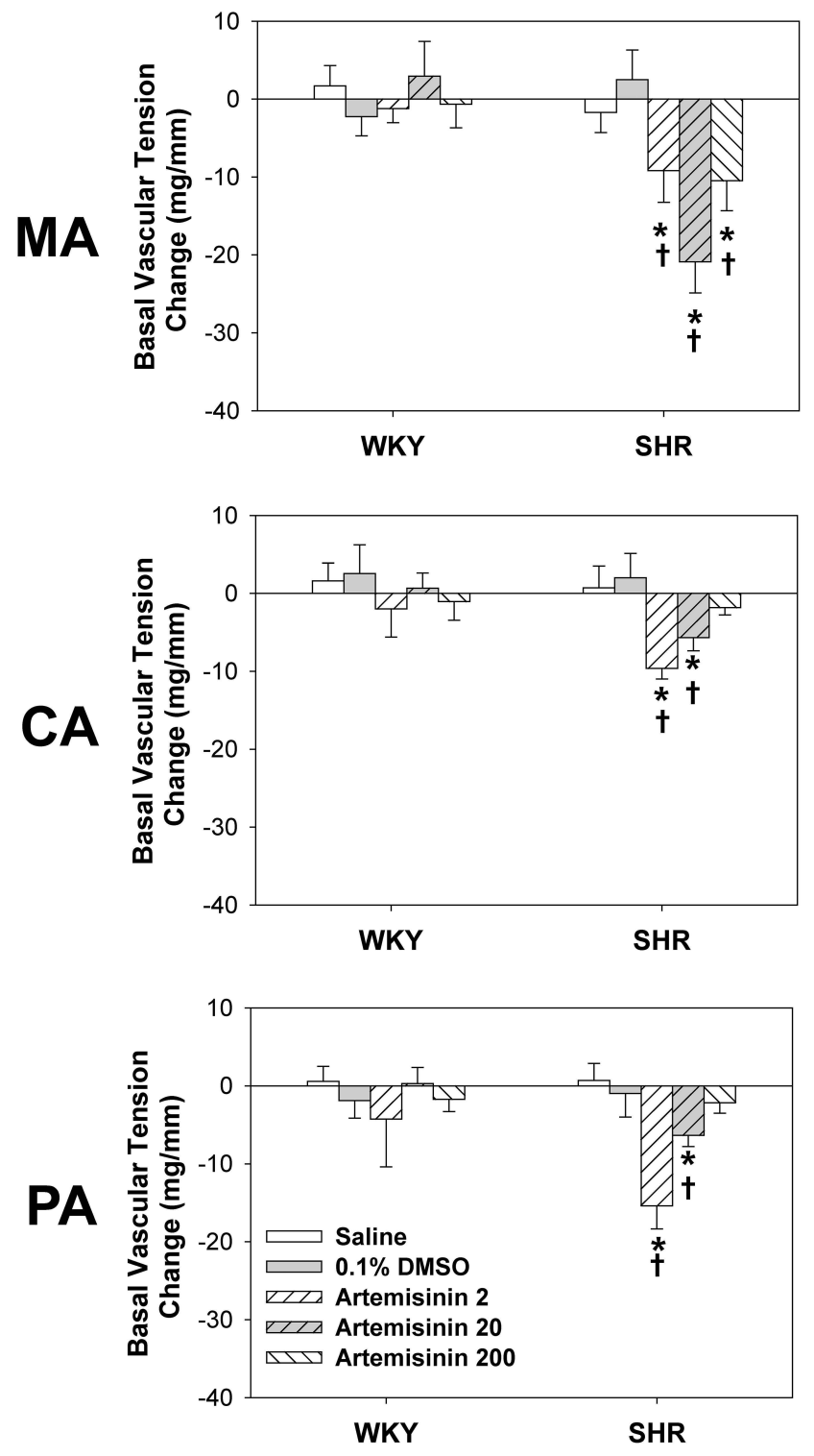

Figure 2 The effects of incubation with saline, 0.1\% DMSO, and artemisinin (2, 20, $200 \mu \mathrm{mol} / \mathrm{L}$ ) on the basal vascular tension of the mesenteric artery (MA), pulmonary artery (PA) and coronary artery (CA) from WKYs and SHRs. Data represent mean \pm SE. $* P<0.05$ compared to saline or DMSO; ${ }^{\dagger} P<0.05$ compared to WKY; $n=6$ for each group.

WKYs. Compared to WKY, ACh-induced dose-dependent relaxations in the $\mathrm{MA}, \mathrm{CA}$ and $\mathrm{PA}$ from SHRs were significantly attenuated. All three doses of artemisinin improved the attenuation of endothelium-dependent vascular relaxation of $\mathrm{MA}, \mathrm{CA}$ and PA from SHRs. There were no significant differences among the effects induced by the three doses of artemisinin on MA or CA from SHRs. For PA from SHRs, the improving effect of the low dose of artemisinin was the best (Figure 3). Analysis of the pD2 (- $\operatorname{logEC} 50)$ and Emax data of the ACh-induced dose-dependent vasodilatation curves of MA, CA and PA from the WKYs and SHRs for each treatment are shown in Table 2, which also supported the above findings.

\section{Effects of Artemisinin on High $\mathrm{K}^{+}$ Solution-Induced Vasoconstriction in WKYs and SHRs}

To investigate artemisinin's role in vasoconstriction, pretreatment of MA, CA or PA with artemisinin $(20 \mu \mathrm{mol} / \mathrm{L})$ was performed for one hour before high $\mathrm{K}^{+}$solutioninduced vasoconstriction. We found that high $\mathrm{K}^{+}$solutioninduced vasoconstriction was enhanced in the MA, CA and PA from SHRs compared to those from WKYs. However, artemisinin pretreatment had no significant effect on high $\mathrm{K}^{+}$solution-induced vasoconstriction of arteries from either WKYs or SHRs (Table 3), which indicates that artemisinin does not influence vascular smooth muscle cell contractile function acutely.

\section{Effects of Artemisinin on eNOS Activity and NO Levels in Arteries from WKYs and SHRs}

To further investigate the downstream molecular mechanisms of artemisinin in regulating endothelial function, the effects of artemisinin on eNOS activity and NO levels in arteries from WKYs and SHRs were measured. Artemisinin incubation had no significant effect on eNOS activity (Figure 4A) or NO levels (Figure 4B) in MA, CA and PA from WKYs. For SHRs, both the eNOS activity and the NO levels in the arteries were lower than those in the WKYs, suggesting impairment of the eNOS-NO system in SHRs. After incubation with artemisinin, the eNOS activity and NO levels of all three arteries from the SHRs recovered to nearly normal levels (Figure 4).

\section{Effects of Artemisinin on NAD(P)H Oxidase Activity and Superoxide Anion Levels in WKY and SHR Arteries}

To investigate whether ROS were involved in the downstream molecular mechanisms of artemisinin, the effects of artemisinin on $\mathrm{NAD}(\mathrm{P}) \mathrm{H}$ oxidase activity and superoxide anion levels in arteries from WKYs and SHRs were detected. Artemisinin treatment had no significant effects on the $\mathrm{NAD}(\mathrm{P}) \mathrm{H}$ oxidase activity (Figure $5 \mathrm{~A}$ ) or the superoxide anion levels (Figure 5B) in MA, CA and PA from WKYs. For SHRs, NAD(P)H oxidase activity and ROS production in the three arteries were higher than those from 

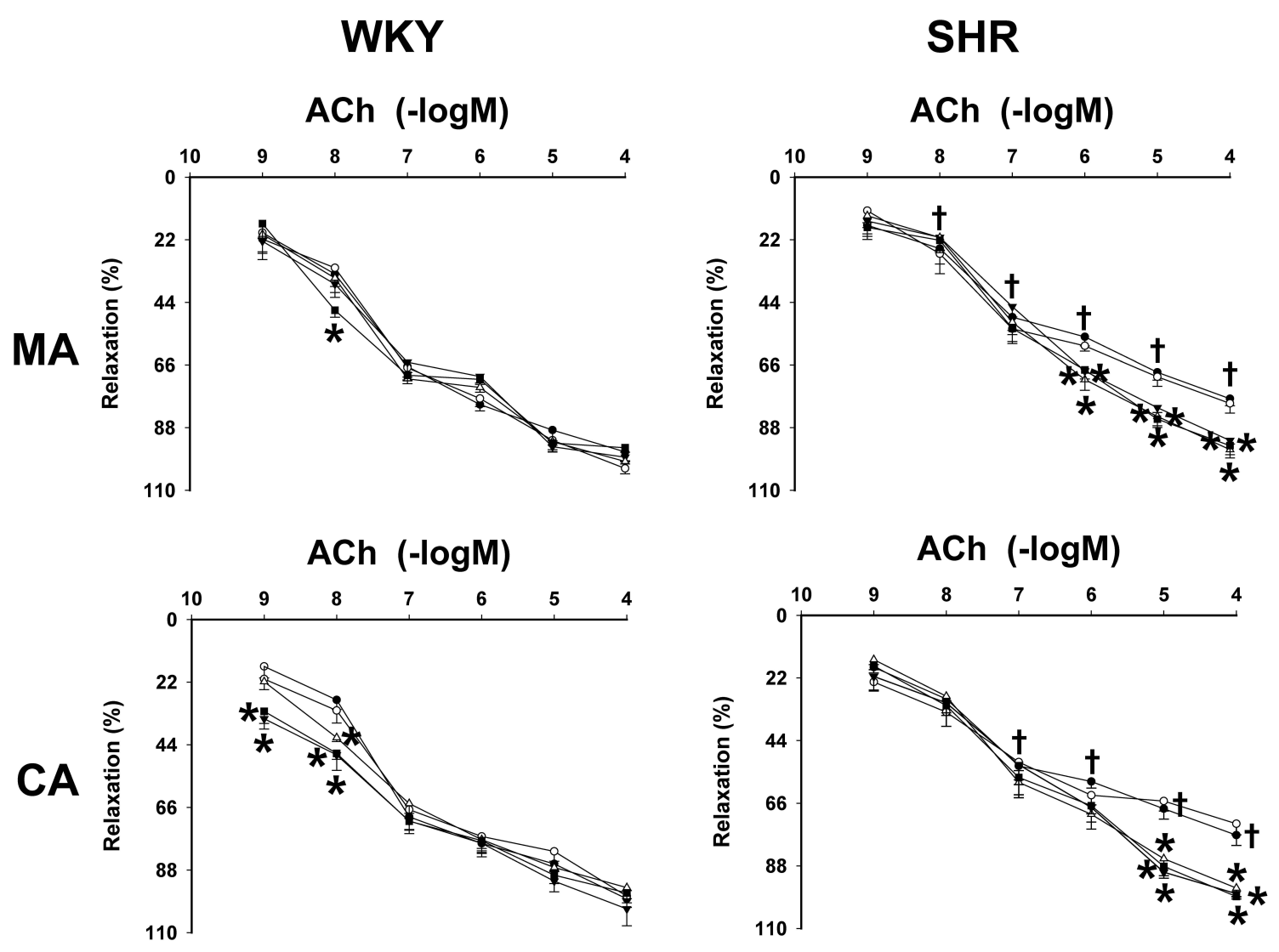

\section{ACh $(-\log M)$}
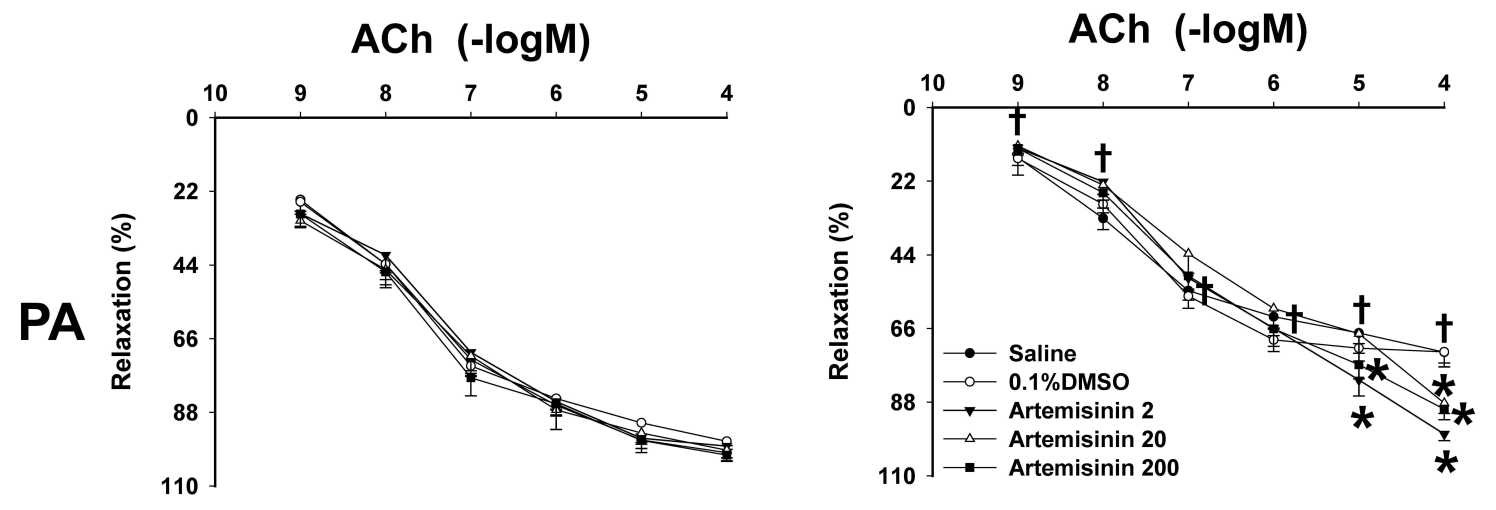

Figure 3 The effects of incubation with saline, 0.1\% DMSO, and artemisinin (2, 20, $200 \mu \mathrm{mol} / \mathrm{L})$ on acetylcholine (ACh)-induced dose-dependent relaxation in the mesenteric artery (MA), pulmonary artery (PA) and coronary artery (CA) from WKYs and SHRs. Data represent mean \pm SE. $* P<0.05$ compared to saline or $D M S O$; ${ }^{\dagger} P<0.05$ $\mathrm{SHR}+$ saline compared to $\mathrm{WKY}+$ saline; $\mathrm{n}=6$ for each group.

the WKYs. These abnormalities were significantly reversed in response to artemisinin treatment of SHRs (Figure 5).

\section{Effects of Chronic Application of Artemisinin to WKYs and SHRs}

To further clarify the long-term effects of artemisinin on arterial blood pressure and endothelial function, chronic application of artemisinin by intraperitoneal injection daily for 28 days was performed. Chronic application of artemisinin had no significant effect on systolic blood pressure
(Figure 6A), mean arterial pressure (Figure 6B), heart rate (Figure 6C), ACh- (Figure 7A) or sodium nitroprusside (Figure 7B)-induced dose-dependent relaxation in WKYs. In SHRs, although chronic application of artemisinin failed to affect the systolic blood pressure and mean arterial pressure, it still decreased the heart rate (Figure 6C), consistent with the results of the acute artemisinin intravenous injection experiment. As the $\mathrm{NAD}(\mathrm{P}) \mathrm{H}$ oxidase subunits NOX-2 and NOX-4 are major sources of ROS in the vascular wall, ${ }^{34-36}$ we tested NOX-2 (Figure 6E) 
Table 2 The pD2 (-logEC50) and Emax (\%) Data of ACh-Induced Dose-Dependent Vasodilatation of Each Group of Treatments on $M A, C A$ and PA in WKYs and SHRs

\begin{tabular}{|c|c|c|c|c|}
\hline & \multicolumn{2}{|c|}{ WKY } & \multicolumn{2}{|c|}{ SHR } \\
\hline & pD2 & Emax & pD2 & Emax \\
\hline \multicolumn{5}{|l|}{ MA } \\
\hline Saline & $7.33 \pm 0.21$ & $96.5 \pm 3.43$ & $7.11 \pm 0.28$ & $77.8 \pm 2.43^{\dagger}$ \\
\hline $0.1 \% \mathrm{DMSO}$ & $7.27 \pm 0.15$ & $102.3 \pm 1.91$ & $7.13 \pm 0.26$ & $79.5 \pm 3.4 \mathrm{I}^{\dagger}$ \\
\hline Artemisinin $2 \mathrm{umol} / \mathrm{L}$ & $7.67 \pm 0.29$ & $98.4 \pm 1.22$ & $6.83 \pm 0.25$ & $92.5 \pm 3.33 *$ \\
\hline Artemisinin $20 \mathrm{umol} / \mathrm{L}$ & $7.76 \pm 0.20$ & $99.9 \pm 0.87$ & $7.34 \pm 0.61$ & $95.7 \pm 2.89 *$ \\
\hline Artemisinin $200 \mathrm{umol} / \mathrm{L}$ & $7.73 \pm 0.23$ & $95.1 \pm 1.55$ & $7.14 \pm 0.31$ & $94.3 \pm 3.16 *$ \\
\hline DMSO-chronic Application & $7.19 \pm 0.20$ & $100.0 \pm 0.97$ & $7.25 \pm 0.35$ & $78.5 \pm 1.07^{\dagger}$ \\
\hline Artemisinin-chronic Application & $7.26 \pm 0.27$ & $97.7 \pm 0.87$ & $7.44 \pm 0.11$ & $97.6 \pm 0.62 *$ \\
\hline \multicolumn{5}{|l|}{ CA } \\
\hline Saline & $7.51 \pm 0.29$ & $98.1 \pm 1.43$ & $7.4 I \pm 0.31$ & $77.2 \pm 3.60^{\dagger}$ \\
\hline $0.1 \% \mathrm{DMSO}$ & $7.66 \pm 0.38$ & $97.2 \pm 3.94$ & $7.52 \pm 0.22$ & $73.2 \pm 4.88^{\dagger}$ \\
\hline Artemisinin 2 umol/L & $7.84 \pm 0.13$ & $101.7 \pm 5.93$ & $7.22 \pm 0.28$ & $97.8 \pm 1.11 *$ \\
\hline Artemisinin $20 \mathrm{umol} / \mathrm{L}$ & $7.82 \pm 0.47$ & $94.2 \pm 1.05$ & $7.40 \pm 0.42$ & $95.8 \pm 5.03 *$ \\
\hline Artemisinin $200 \mathrm{umol} / \mathrm{L}$ & $7.58 \pm 0.37$ & $96.1 \pm 2.04$ & $7.27 \pm 0.44$ & $98.8 \pm 0.81^{*}$ \\
\hline DMSO-chronic Application & $7.5 I \pm 0.20$ & $98.4 \pm 3.07$ & $7.16 \pm 0.27$ & $77.1 \pm 1.50^{\dagger}$ \\
\hline Artemisinin-chronic Application & $7.48 \pm 0.14$ & $97.6 \pm 1.94$ & $7.19 \pm 0.25$ & $91.5 \pm 3.47 *$ \\
\hline \multicolumn{5}{|l|}{ PA } \\
\hline Saline & $7.54 \pm 0.43$ & $100.0 \pm 2.63$ & $7.37 \pm 0.51$ & $73.1 \pm 3.25^{\dagger}$ \\
\hline $0.1 \% \mathrm{DMSO}$ & $7.7 I \pm 0.35$ & $96.7 \pm 1.29$ & $7.50 \pm 0.47$ & $77.0 \pm 4.45^{\dagger}$ \\
\hline Artemisinin 2 umol/L & $7.31 \pm 0.28$ & $98.1 \pm 0.92$ & $6.98 \pm 0.19$ & $97.5 \pm 1.96 *$ \\
\hline Artemisinin $20 \mathrm{umol} / \mathrm{L}$ & $7.42 \pm 0.44$ & $99.3 \pm 0.84$ & $6.99 \pm 0.24$ & $88.3 \pm 5.13 *$ \\
\hline Artemisinin $200 \mathrm{umol} / \mathrm{L}$ & $7.70 \pm 0.26$ & $100.7 \pm 0.88$ & $7.31 \pm 0.53$ & $88.1 \pm 5.15^{*}$ \\
\hline DMSO-chronic Application & $7.44 \pm 0.11$ & $95.8 \pm 1.72$ & $7.38 \pm 0.04$ & $78.8 \pm 3.11^{\dagger}$ \\
\hline Artemisinin-chronic Application & $7.46 \pm 0.11$ & $93.3 \pm 1.55$ & $8.03 \pm 0.49$ & $89.6 \pm 1.40 *$ \\
\hline
\end{tabular}

Notes: Values are mean \pm SE. ${ }^{*} \mathrm{P}<0.05$ vs Saline or $0.1 \% D M S O$ or DMSO-chronic Application. ${ }^{\dagger} \mathrm{P}<0.05$ vs $\mathrm{WKY}$ s. $\mathrm{n}=6$ for each group.

Table 3 Effects of Artemisinin $(20 \mu \mathrm{mol} / \mathrm{L})$ on the High $\mathrm{K}^{+}$Solution Induced Contraction $(\mathrm{mg} / \mathrm{mm})$ in MA, CA and PA in WKYs and SHRs

\begin{tabular}{|l|c|c|c|c|c|c|}
\hline \multirow{2}{*}{} & \multicolumn{3}{|c|}{ WKY } & \multicolumn{3}{c|}{ SHR } \\
\cline { 2 - 7 } & Saline & 0.1\%DMSO & Artemisinin & Saline & 0.1\%DMSO & Artemisinin \\
\hline MA & $258.2 \pm 19.8$ & $271.3 \pm 23.1$ & $266.4 \pm 26.4$ & $665.5 \pm 28.6^{\dagger}$ & $674.2 \pm 25.1^{\dagger}$ & $669.7 \pm 31.4^{\dagger}$ \\
CA & $157.5 \pm 9.8$ & $161.5 \pm 36.2$ & $168.2 \pm 28.7$ & $295.6 \pm 25.5^{\dagger}$ & $305.7 \pm 30.2^{\dagger}$ & $290.0 \pm 26.7^{\dagger}$ \\
PA & $203.5 \pm 20.4$ & $199.3 \pm 11.9$ & $223.1 \pm 21.0$ & $346.7 \pm 20.4^{\dagger}$ & $339.6 \pm 26.9^{\dagger}$ & $323.0 \pm 20.2^{\dagger}$ \\
\hline
\end{tabular}

Notes: Values are expressed as mean \pm SE. ${ }^{*} P<0.05$ vs Saline or $0.1 \% D M S O{ }^{\dagger} P<0.05$ vs $W K Y s . n=6$ for each group.

and NOX-4 (Figure 6F) protein expression together with eNOS (Figure 6D) in the MA of rats after chronic application of artemisinin for 28 days. Compared with WKYs, the protein expression of eNOS was decreased, while the NOX-2 and NOX-4 protein expression levels were increased in SHRs, which was reversed by chronic application of artemisinin. Furthermore, after chronic application of artemisinin, the attenuated ACh-induced endothelium-dependent relaxation in SHRs was also improved (Figure 7A). There was no significant difference in sodium nitroprusside-induced dose-dependent relaxation between WKYs and SHRs, and chronic application of artemisinin had no significant effect on sodium nitroprusside-induced endothelium-independent relaxation in 
MA

A

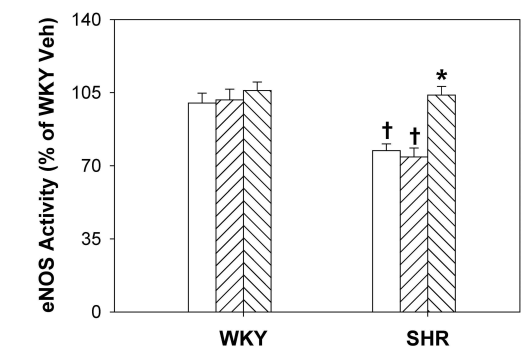

B

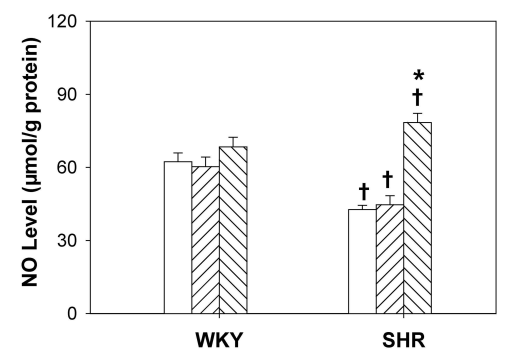

CA
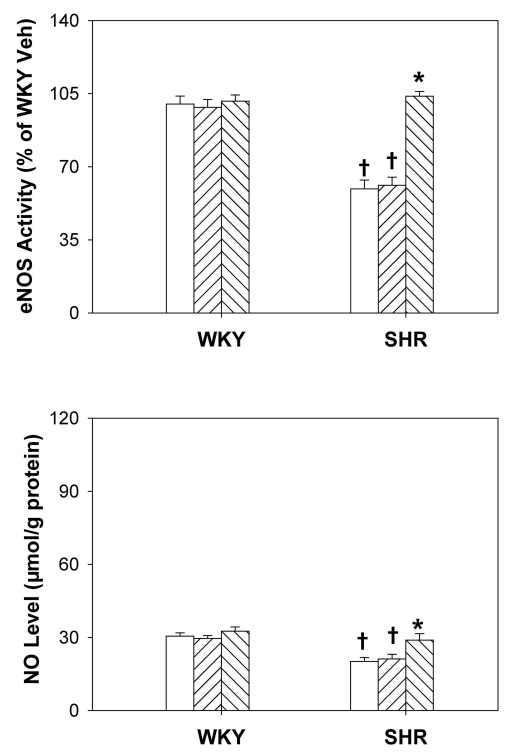

PA
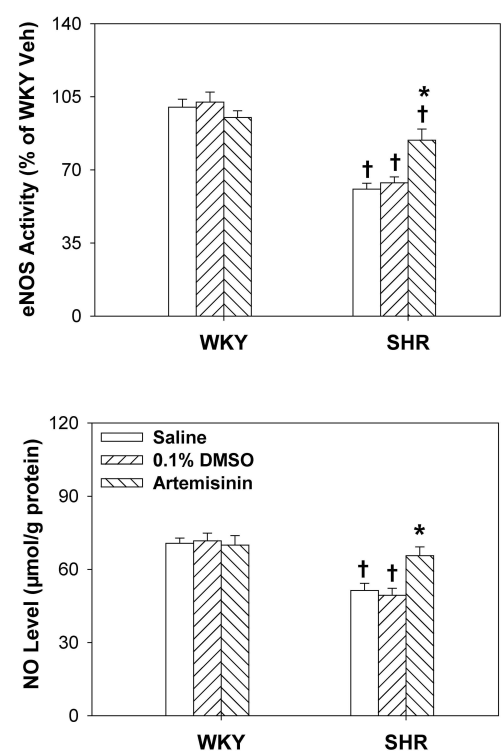

Figure 4 The effects of incubation with saline, $0.1 \%$ DMSO, and artemisinin $(20 \mu \mathrm{mol} / \mathrm{L})$ on endothelial nitric oxide (NO) synthase (eNOS) activity (A) and NO levels (B) of the mesenteric artery (MA), pulmonary artery $(\mathrm{PA})$ and coronary artery $(\mathrm{CA})$ from WKYs and SHRs. Data represent mean $\pm \mathrm{SE}$. $* \mathrm{P}<0.05$ compared to saline or DMSO; ${ }^{\dagger} P<0.05$ compared to $\mathrm{WKY} ; \mathrm{n}=6$ for each group.

A

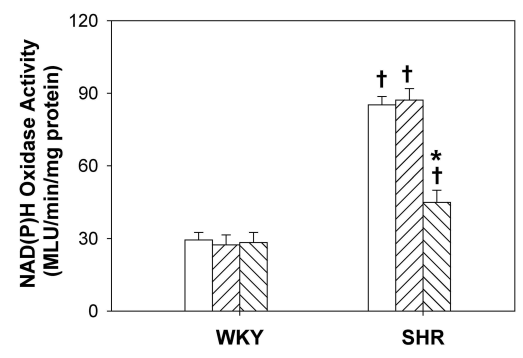

B

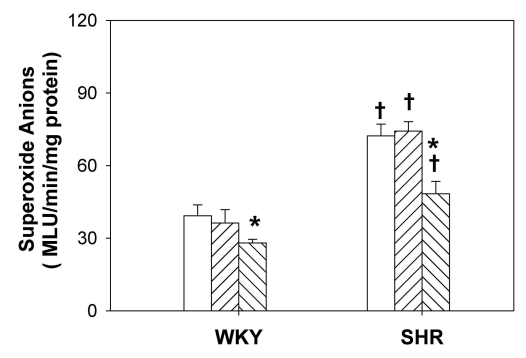

CA
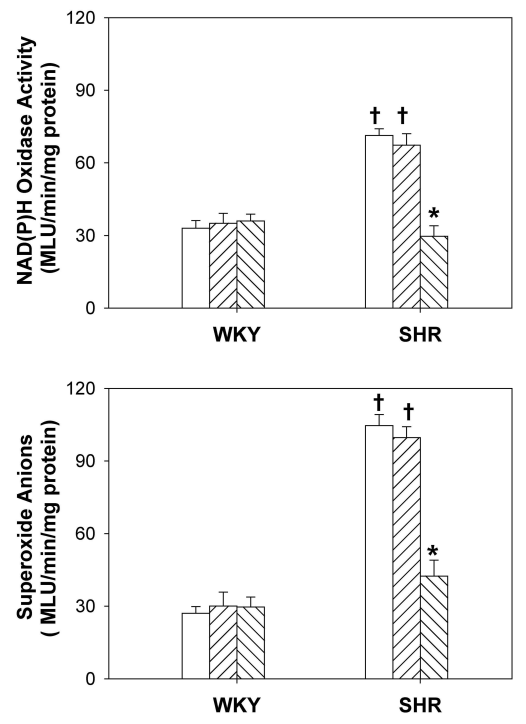

PA
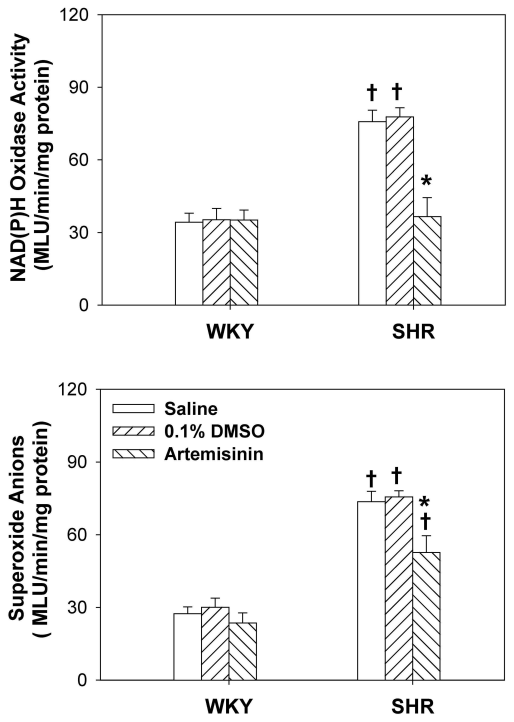

Figure 5 The effects of incubation with saline, $0.1 \%$ DMSO, and artemisinin $(20 \mu \mathrm{mol} / \mathrm{L})$ on NAD(P)H oxidase activity (A) and superoxide anion levels (B) of the mesenteric artery (MA), pulmonary artery (PA) and coronary artery (CA) from WKYs and SHRs. Data represent mean \pm SE. $* P<0.05$ compared to saline or DMSO; ${ }^{\dagger} P<0.05$ compared to WKY; $n=6$ for each group.

SHRs (Figure 7B), which eliminated artemisinin's function in vascular smooth muscle cell relaxation. Table 2 shows the pD2 and Emax data of ACh-induced dosedependent vasodilatation of MA, CA and PA from WKYs and SHRs after chronic application of artemisinin or DMSO.

\section{Discussion}

Increased vascular constriction and attenuated vascular relaxation of small arteries due to endothelial dysfunction are major causes of the persistent increase in total peripheral resistance leading to the progression of organ damage and the development of hypertension. ${ }^{37,38}$ The contraction 
A
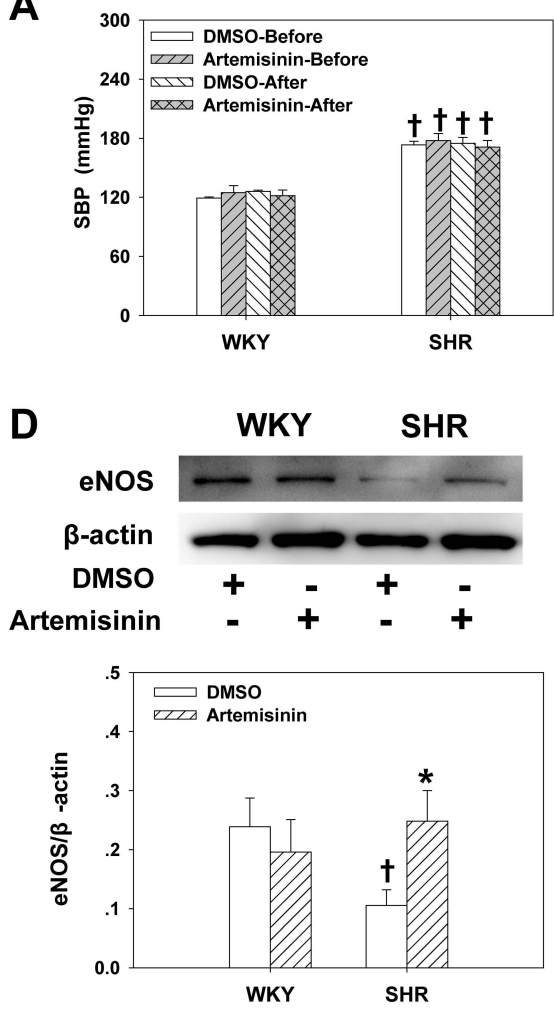

B

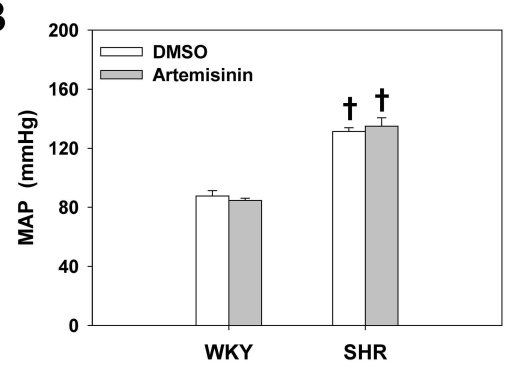

E
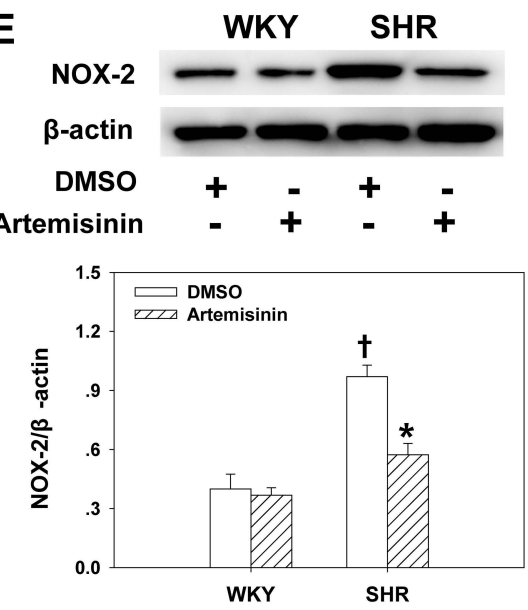

C

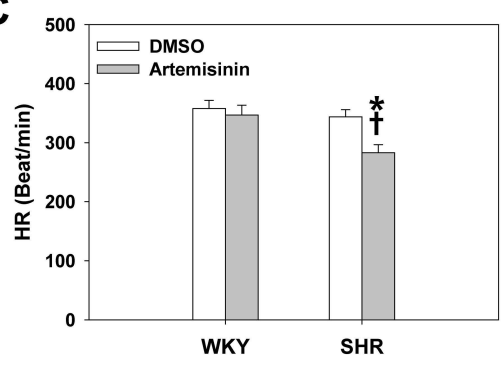

F
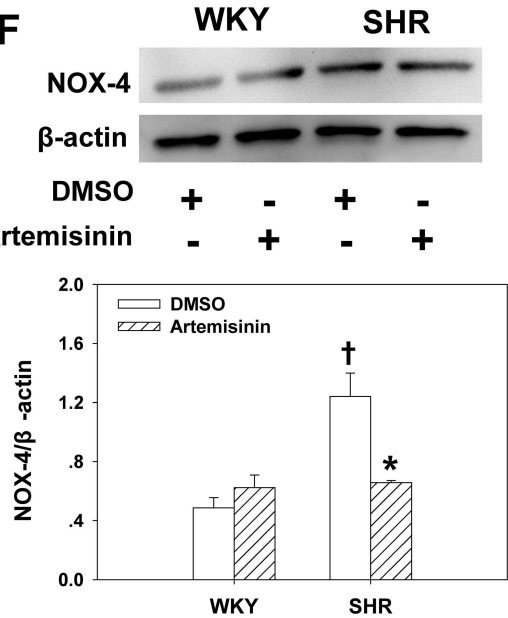

Figure 6 The effects of chronic application of artemisinin and DMSO by intraperitoneal injection for 28 days on systolic blood pressure (SBP) (A) in the conscious state, mean arterial pressure (MAP) (B) and heart rate (HR) (C) under anaesthesia, and the protein expression of endothelial nitric oxide synthase (eNOS) (D), NAD(P)H oxidase subunits NOX-2 (E) and NOX-4 (F) of the mesenteric artery from WKYs and SHRs. Data represent mean \pm SE. $* P<0.05$ compared to DMSO; ${ }^{\dagger} P<0.05$ compared to WKY; $n=6$ for the A-C group; $n=3$ for the $D-F$ group.

\section{MA}

A

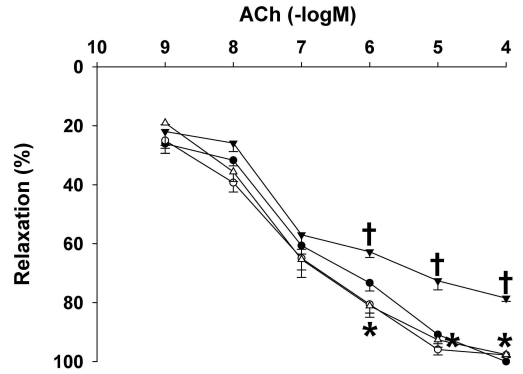

SNP $(-\log M)$

B

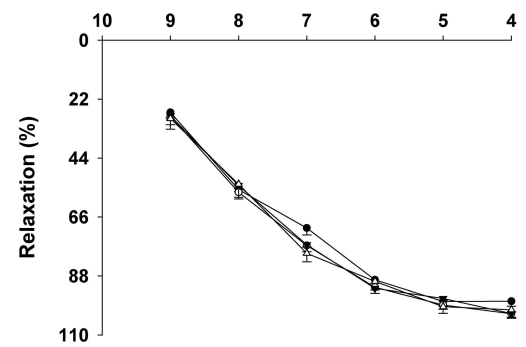

CA

ACh (-logM)

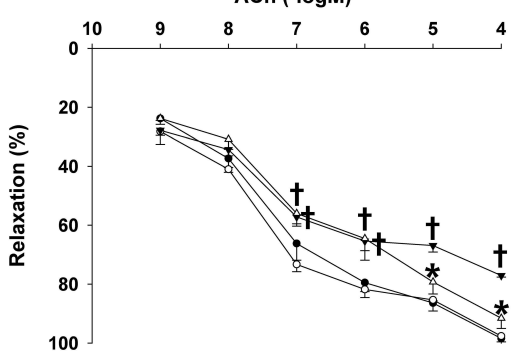

SNP (-logM)

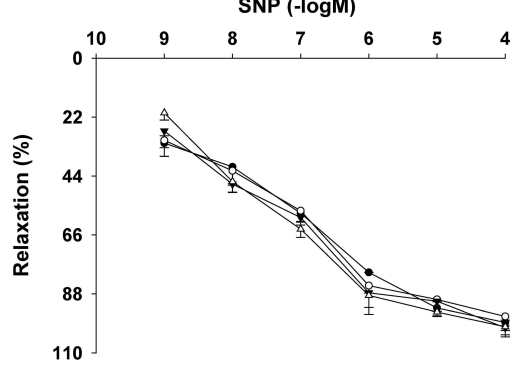

PA
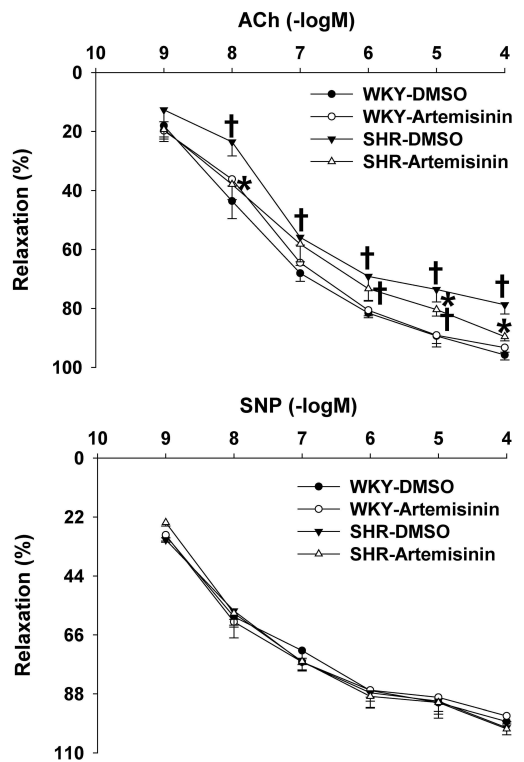

Figure 7 The effects of chronic application of artemisinin and DMSO on acetylcholine (ACh) (A) or sodium nitroprusside (SNP) (B)-induced dose-dependent relaxations of the mesenteric artery (MA), pulmonary artery (PA) and coronary artery (CA) from WKYs and SHRs. Data represent mean \pm SE. $* \mathrm{P}<0.05$ compared to DMSO; ${ }^{\dagger} \mathrm{P}<0.05$ compared to WKY; $\mathrm{n}=6$ for each group. 
or relaxation of enormous amounts of mesenteric arteries is directly connected to the total peripheral resistance and it influences arterial blood pressure. ${ }^{39}$ Due to attenuated vasodilatation and sustained coronary arterial contraction, coronary artery disease is a typical cause of mortality in hypertensive patients. $^{40,41}$ Attenuated pulmonary arterial relaxation increases pulmonary vascular resistance and the risk of pulmonary hypertension in hypertensive patients. ${ }^{42-44}$ It has been reported that artemisinin and its derivatives reduce the risk of cardiovascular diseases partly by protecting the vascular endothelium from injury and enhancing the activation of eNOS in endothelial cells. ${ }^{15,19,25}$ However, the effect of artemisinin on endothelial dysfunction in hypertension remains unknown. Therefore, in this study, we used the mesenteric artery, coronary artery and pulmonary artery, three important arteries, to determine the effects of artemisinin on endothelial function and vascular relaxation in primary hypertension. We are the first to show that artemisinin is able to improve endothelium-dependent vascular relaxation and decrease the basal vascular tension of the mesenteric artery, coronary artery and pulmonary artery in primary hypertension. We also demonstrated that artemisinin is able to increase eNOS activity and NO levels and decrease $\mathrm{NAD}(\mathrm{P}) \mathrm{H}$ oxidase activity and superoxide anion levels in the arteries of spontaneously hypertensive rats. These results suggest that artemisinin might play important roles in the treatment of endothelial dysfunction in primary hypertension.

Previous studies have reported that artemisinin and its derivatives exert protective roles in cardiovascular disease via their anti-inflammatory, antifibrotic, antiproliferation and antimigration effects in addition to their antimalarial activity. Artemisinin alleviates atherosclerotic lesions in high-fat diet-fed ApoE-/- mice by promoting macrophage autophagy through the AMPK/mTOR/ULK1 pathway or by modulating vascular smooth muscle cell phenotype switching. ${ }^{11,12}$ Artemisinin inhibits the proliferation, migration, and inflammatory reactions induced by tumour necrosis factor-alpha in vascular smooth muscle cells through the nuclear factor-kappa B (NF- $\kappa \mathrm{B})$ pathway. ${ }^{18}$ Artemisinin also protects rats against cardiac hypertrophy in vivo and blocks angiotensin II-induced cardiac hypertrophy in a concentration-dependent manner in vitro by interfering with NF- $\mathrm{KB}$ signalling. ${ }^{13}$ Dihydroartemisinin attenuates pulmonary hypertension via inhibition of pulmonary vascular remodelling in rats. ${ }^{14,15}$ Delivering artemether locally into the subcutaneous fat or through the tail vein in mice was found to reduce high-fat diet-induced body weight gain, improve insulin sensitivity and enhance cold tolerance. ${ }^{16,17}$ In diet-induced obese mice, artemisinin attenuates hepatic steatosis and inflammation. ${ }^{16,17}$ However, the effect of artemisinin treatment in hypertension remains uncertain. Interestingly, some investigators have linked malaria to the growing burden of hypertension. Studies have reported that malaria parasitemia and hypertension are seemingly linked comorbidities. ${ }^{45,46}$ Therefore, it is important to clarify the roles of artemisinin in hypertension.

It has been reported that dihydroartemisinin has an antihypoxic effect on pulmonary artery endothelial cells and plays an important inhibitory role in regulating the $\mathrm{PGE}_{2}$ synthesis cascade and inflammation in endothelial cells. ${ }^{15}$ Artesunate has a protective effect on lipopolysaccharide-induced human umbilical vein endothelial cell injury $^{19}$ and it remarkably inhibits the proliferation and differentiation of endothelial cells in a dose-dependent manner. ${ }^{23}$ In the present study, we found that compared to WKYs, ACh-induced endothelium-dependent relaxation of arteries from SHRs was significantly attenuated, suggesting an imbalance between vasodilator and vasoconstrictor factor release from endothelial cells and the occurrence of endothelial dysfunction in SHRs. Artemisinin incubation significantly improved endothelium-dependent vascular relaxation and decreased basal vascular tension of the MA, CA and PA from SHRs but had no significant effect on high $\mathrm{K}^{+}$solution-induced vasoconstriction of arteries in either WKYs or SHRs. After chronic application of artemisinin for 28 days, AChinduced endothelium-dependent relaxation of MA, CA and PA from SHRs was also improved, while there was no significant change in sodium nitroprusside-induced endothelium-independent relaxation in arteries from SHRs. These results indicated that artemisinin has beneficial effects in improving vascular endothelial function to increase vascular relaxation and prevent endothelial dysfunction in primary hypertension, but it plays no role in influencing vascular smooth muscle cell contractile and diastolic function. Furthermore, we speculated that the decrease in heart rate response to artemisinin application might be a consequence of the improvement of coronary artery endothelium-dependent relaxation, which increased the blood supply to the myocardium and improved the cardiac function.

The mechanisms of the protective or improving effects of artemisinin on endothelial function remain uncertain. It 
has been reported that treatment with artesunate enhances the phosphorylation of eNOS in haemorrhagic shock rats. ${ }^{25}$ Artesunate also mitigates hypoxia/reoxygenationmediated increases in ROS levels in alveolar macrophages. ${ }^{26}$ In this study, we found that eNOS activity, eNOS protein level and NO production of arteries isolated from SHRs were significantly lower, while NAD(P)H oxidase activity, $\mathrm{NAD}(\mathrm{P}) \mathrm{H}$ oxidase subunits $\mathrm{NOX}-2$ and NOX-4 protein expression and superoxide anion levels were higher than those in arteries from WKYs, which indicated the occurrence of heavy oxidative stress and a decrease in eNOS-NO bioavailability in arteries with primary hypertension. Treatment of arteries with artemisinin significantly decreased NAD(P)H oxidase activity and superoxide anion levels and increased eNOS activity and NO production in the MA, CA and PA from SHRs. Chronic administration of artemisinin to SHRs also increased the protein expression of eNOS and decreased NOX-2 and NOX-4 protein expression. These results suggest that artemisinin has the ability to inhibit $\mathrm{NAD}(\mathrm{P}) \mathrm{H}$ oxidase-derived ROS production and increase eNOS activation to release NO in endothelial cells. We think that because of this important mechanism, artemisinin could improve endothelium-dependent vascular relaxation and decrease basal vascular tension of the mesenteric artery, coronary artery and pulmonary artery and then play a beneficial role in decreasing the total peripheral resistance, increasing the blood supply to the heart and decreasing the pulmonary vascular resistance and the risk of pulmonary hypertension in hypertensive statues.

We found that artemisinin represents a pharmacological therapy for endothelial dysfunction of all three small arteries in SHRs, but the effects of artemisinin were not dosedependent. It is possible that the high dose of artemisinin that we used was supra-maximal and caused some side effects, which is a limitation of the present study and needs to be verified in the future. In addition, it is unexpected that both acute intravenous application of artemisinin via the jugular vein and chronic intraperitoneal administration of artemisinin had no significant effect on the blood pressure of SHRs. We think this might be due to the vascular tension test being an isolated blood vessel test, while animal blood pressure in vivo is regulated by a more complicated system, including neuromodulation, such as sympathetic or parasympathetic nerve regulation, ${ }^{47,48}$ and humoral regulation, such as epinephrine ${ }^{49}$ and angiotensin $\mathrm{II}^{50}$ regulation. On the other hand, artemisinin might also play other roles inside the body that we do not yet comprehend. This is another limitation of the present study and should be explored in the future.

In conclusion, this study found that artemisinin decreases the heart rate and basal vascular tension and improves attenuated endothelium-dependent vascular relaxation in spontaneous hypertension, which might be implemented by inhibiting $\mathrm{NAD}(\mathrm{P}) \mathrm{H}$ oxidase activityderived ROS generation and increasing eNOS activationNO release by endothelial cells. The results of this study suggest that treatment with artemisinin may partially preserve endothelial function, conveying beneficial effects from pharmacological therapy for endothelial dysfunction during the development and progression of hypertension.

\section{Abbreviations}

ACh, acetylcholine; CA, coronary artery; eNOS, endothelial nitric oxide synthase; MA, mesenteric artery; NO, nitric oxide; PA, pulmonary artery; ROS, reactive oxygen species; SHR, spontaneously hypertensive rat; WKY, Wistar-Kyoto rat.

\section{Data Sharing Statement}

The data that support the findings of this study are available from the corresponding author Ying Han upon reasonable request.

\section{Ethical Approval}

All applicable international, national and/or institutional guidelines for the care and use of animals were followed. All animal procedures were reviewed and approved by Nanjing Medical University Experimental Animal Care committee and in accordance with the Guide for the Care and Use of Laboratory Animals published by the US National Institutes of Health (NIH publication, 8th edition, 2011).

\section{Funding}

This work was sponsored by the National Natural Science Foundation of China [81470538, 81970052 and 82170057], Qing Lan Project of Jiangsu Province of China and the Open Project of the State Key Laboratory of Respiratory Disease (SKLRD-OP-201911).

\section{Disclosure}

The authors report no conflicts of interest in this work.

\section{References}

1. Panieri E, Santoro MM. ROS signaling and redox biology in endothelial cells. Cell Mol Life Sci. 2015;72(17):3281-3303. 
2. Higashi Y, Sasaki S, Nakagawa K, Matsuura H, Oshima T, Chayama K. Endothelial function and oxidative stress in renovascular hypertension. $N$ Engl J Med. 2002;346(25):1954-1962. doi:10.1056/ NEJMoa013591

3. Doyle AE. Hypertension and vascular disease. Am J Hypertens. 1991;4(2 Pt 2):103S-106S. doi:10.1093/ajh/4.2.103S

4. Ghimire K, Altmann HM, Straub AC, Isenberg JS. Nitric oxide: what's new to NO? Am J Physiol Cell Physiol. 2017;312(3):C254 C262. doi:10.1152/ajpcell.00315.2016

5. Raffetto JD, Calanni F, Mattana P, Khalil RA. Sulodexide promotes arterial relaxation via endothelium-dependent nitric oxide-mediated pathway. BiochemPharmacol. 2019;166:347-356.

6. Araujo AV, Andrade FA, Paulo M, et al. NO donors induce vascular relaxation by different cellular mechanisms in hypertensive and normotensive rats. Nitric Oxide. 2019;86:12-20. doi:10.1016/j.niox. 2019.02.004

7. Zhang F, Tang H, Sun S, et al. Angiotensin-(1-7) induced vascular relaxation in spontaneously hypertensive rats. Nitric Oxide. 2019;88:1-9. doi:10.1016/j.niox.2019.03.007

8. Zhang F, Xu Y, Pan Y, et al. Effects of angiotensin-(1-7) and angiotensin II on acetylcholine-induced vascular relaxation in spontaneously hypertensive rats. Oxid Med Cell Longev. 2019;20 19:6512485. doi:10.1155/2019/6512485

9. Jiang W, Cen Y, Song Y, et al. Artesunate attenuated progression of atherosclerosis lesion formation alone or combined with rosuvastatin through inhibition of pro-inflammatory cytokines and pro-inflammatory chemokines. Phytomedicine. 2016;23(11):1259-1266. doi:10.1016/j. phymed.2016.06.004

10. Wang HY, Huang RP, Han P, et al. The effects of artemisinin on the proliferation and apoptosis of vascular smooth muscle cells of rats. Cell Biochem Funct. 2014;32(2):201-208.

11. Cao Q, Du H, Fu X, Duan N, Liu C, Li X. Artemisinin attenuated atherosclerosis in high-fat diet-fed ApoE-/- mice by promoting macrophage autophagy through the AMPK/mTOR/ULK1 pathway. J Cardiovasc Pharmacol. 2020;75(4):321-332. doi:10.1097/ FJC.0000000000000794

12. Du H, Zhao Q, Zang H, Chang C, Li X. Artemisinin attenuates the development of atherosclerotic lesions by the regulation of vascular smooth muscle cell phenotype switching. Life Sci. 2019;237:116943. doi:10.1016/j.1fs.2019.116943

13. Xiong Z, Sun G, Zhu C, et al. Artemisinin, an anti-malarial agent, inhibits rat cardiac hypertrophy via inhibition of NF-kappaB signaling. Eur J Pharmacol. 2010;649(1-3):277-284. doi:10.1016/j. ejphar.2010.09.018

14. Tang M, Wang R, Feng P, et al. Dihydroartemisinin attenuates pulmonary hypertension through inhibition of pulmonary vascular remodeling in rats. $J$ Cardiovasc Pharmacol. 2020;76(3):337-348. doi:10.1097/FJC.0000000000000862

15. Yu H, Liu J, Dong Y, et al. Anti-hypoxic effect of dihydroartemisinin on pulmonary artery endothelial cells. Biochem Biophys Res Commun. 2018;506(4):840-846. doi:10.1016/j.bbrc.2018.10.176

16. Kim KE, Ko KH, Heo RW, et al. Artemisia annua leaf extract attenuates hepatic steatosis and inflammation in high-fat diet-fed mice. J Med Food. 2016;19(3):290-299. doi:10.1089/jmf. 2015.3527

17. Lu P, Zhang FC, Qian SW, et al. Artemisinin derivatives prevent obesity by inducing browning of WAT and enhancing BAT function. Cell Res. 2016;26(10):1169-1172. doi:10.1038/cr.2016.108

18. Cao Q, Jiang Y, Shi J, et al. Artemisinin inhibits the proliferation, migration, and inflammatory reaction induced by tumor necrosis factor-alpha in vascular smooth muscle cells through nuclear factor kappa B pathway. J Surg Res. 2015;194(2):667-678. doi:10.1016/j. jss.2014.12.013

19. He XL, Liu Z. [Protection of artesunate on activation and injury of vascular endothelial cells induced by lipopolysaccharide]. Zhongguo Zhong Xi Yi Jie He Za Zhi. 2004;24(12):1110-1113. Chinese.
20. Dong F, Zhou X, Li C, et al. Dihydroartemisinin targets VEGFR2 via the NF-kappaB pathway in endothelial cells to inhibit angiogenesis. Cancer Biol Ther. 2014;15(11):1479-1488. doi:10.4161/15384047. 2014.955728

21. Zhang Y, Xu G, Zhang S, Wang D, Saravana Prabha P, Zuo Z. Antitumor research on artemisinin and its bioactive derivatives. Nat Prod Bioprospect. 2018;8(4):303-319. doi:10.1007/s13659-0180162-1

22. Efferth T, Romero MR, Wolf DG, Stamminger T, Marin JJ, Marschall M. The antiviral activities of artemisinin and artesunate. Clin Infect Dis. 2008;47(6):804-811. doi:10.1086/591195

23. Huan-huan C, Li-Li Y, Shang-Bin L. Artesunate reduces chicken chorioallantoic membrane neovascularisation and exhibits antiangiogenic and apoptotic activity on human microvascular dermal endothelial cell. Cancer Lett. 2004;211(2):163-173. doi:10.1016/j.canlet.20 04.03.014

24. Lee HA, Kim KS, Kim EJ. General pharmacology of artesunate, a commonly used antimalarial drug: effects on central nervous, cardiovascular, and respiratory system. Toxicol Res. 2010;26 (3):223-232. doi:10.5487/TR.2010.26.3.223

25. Sordi R, Nandra KK, Chiazza F, et al. Artesunate protects against the organ injury and dysfunction induced by severe hemorrhage and resuscitation. Ann Surg. 2017;265(2):408-417. doi:10.1097/SLA.00 00000000001664

26. Liu Z, Qu M, Yu L, Song P, Chang Y. Artesunate inhibits renal ischemia-reperfusion-mediated remote lung inflammation through attenuating ROS-induced activation of NLRP3 inflammasome. Inflammation. 2018;41(4):1546-1556. doi:10.1007/s10753-0180801-Z

27. Sun S, Zhang F, Pan Y, et al. A TOR2A gene product: salusin-beta contributes to attenuated vasodilatation of spontaneously hypertensive rats. Cardiovasc Drugs Ther. 2021;35(1):125-139. doi:10.1007/ s10557-020-06983-1

28. Pan Y, Sun S, Wang X, et al. Improvement of vascular function by knockdown of salusin-beta in hypertensive rats via nitric oxide and reactive oxygen species signaling pathway. Front Physiol. 2021;12:622954. doi:10.3389/fphys.2021.622954

29. Ren XS, Tong Y, Qiu Y, et al. MiR155-5p in adventitial fibroblasts-derived extracellular vesicles inhibits vascular smooth muscle cell proliferation via suppressing angiotensin-converting enzyme expression. $J$ Extracell Vesicles. 2020;9(1):1698795. doi:10.1080/20013078.2019.1698795

30. Han Y, Cho YE, Ayon R, et al. SGLT inhibitors attenuate NO-dependent vascular relaxation in the pulmonary artery but not in the coronary artery. Am J Physiol Lung Cell Mol Physiol. 2015;309 (9):L1027-L1036. doi:10.1152/ajplung.00167.2015

31. Sofola OA, Raji I, Ladipo C, Coker HA. Artesunate causes relaxation of rat aortic rings and reduces the contractile response to noradrenaline. Nig $Q J$ Hosp Med. 2008;18(2):50-52.

32. Tong Y, Liu Y, Zheng $\mathrm{H}$, et al. Artemisinin and its derivatives can significantly inhibit lung tumorigenesis and tumor metastasis through Wnt/beta-catenin signaling. Oncotarget. 2016;7(21):31413-31428. doi:10.18632/oncotarget.8920

33. Sun H, Zhang F, Xu Y, et al. Salusin-beta promotes vascular calcification via nicotinamide adenine dinucleotide phosphate/reactive oxygen species-mediated klotho downregulation. Antioxid Redox Signal. 2019;31(18):1352-1370. doi:10.1089/ars.2019.7723

34. Giacco F, Brownlee M. Oxidative stress and diabetic complications. Circ Res. 2010;107(9):1058-1070. doi:10.1161/CIRCRESAHA. 110.223545

35. Geiszt M. NADPH oxidases: new kids on the block. Cardiovasc Res. 2006;71(2):289-299. doi:10.1016/j.cardiores.2006.05.004

36. Ling WC, Mustafa MR, Vanhoutte PM, Murugan DD. Chronic administration of sodium nitrite prevents hypertension and protects arterial endothelial function by reducing oxidative stress in angiotensin II-infused mice. VasculPharmacol. 2018;102:11-20. 
37. Brandes RP. Endothelial dysfunction and hypertension. Hypertension. 2014;64(5):924-928. doi:10.1161/HYPERTENSIONAHA.114.03575

38. Konukoglu D, Uzun H. Endothelial dysfunction and hypertension. Adv Exp Med Biol. 2017;956:511-540.

39. Chinnathambi V, Balakrishnan M, Ramadoss J, Yallampalli C, Sathishkumar K. Testosterone alters maternal vascular adaptations: role of the endothelial NO system. Hypertension. 2013;61 (3):647-654. doi:10.1161/HYPERTENSIONAHA.111.00486

40. Brush JE Jr, Faxon DP, Salmon S, Jacobs AK, Ryan TJ. Abnormal endothelium-dependent coronary vasomotion in hypertensive patients. J Am Coll Cardiol. 1992;19(4):809-815. doi:10.1016/ 0735-1097(92)90522-O

41. Deussen A, Ohanyan V, Jannasch A, Yin L, Chilian W. Mechanisms of metabolic coronary flow regulation. J Mol Cell Cardiol. 2012;52 (4):794-801. doi:10.1016/j.yjmcc.2011.10.001

42. Fiorentini C, Barbier P, Galli C, et al. Pulmonary vascular overreactivity in systemic hypertension. A pathophysiological link between the greater and the lesser circulation. Hypertension. 1985;7 (6 Pt 1):995-1002. doi:10.1161/01.HYP.7.6.995

43. Gomart S, Damoiseaux C, Jespers $P$, et al. Pulmonary vasoreactivity in spontaneously hypertensive rats-effects of endothelin-1 and leptin. Respir Res. 2014;15:12. doi:10.1186/1465-9921-15-12

44. Aharinejad S, Schraufnagel DE, Bock P, et al. Spontaneously hypertensive rats develop pulmonary hypertension and hypertrophy of pulmonary venous sphincters. Am J Pathol. 1996;148(1):281-290.
45. Eze IC, Bassa FK, Esse C, et al. Epidemiological links between malaria parasitaemia and hypertension: findings from a populationbased survey in rural cote d'Ivoire. J Hypertens. 2019;37 (7):1384-1392. doi:10.1097/HJH.0000000000002071

46. Verdecchia P, Angeli F, Reboldi G. Does malaria cause hypertension? Circ Res. 2016;119(1):7-9. doi:10.1161/CIRCRESAHA.116.309013

47. Robles-Cabrera A, Michel-Chavez A, Callejas-Rojas RC, MalamudKessler C, Delgado G, Estanol-Vidal B. [The cardiovagal, cardiosympathetic and vasosympathetic arterial baroreflexes and the neural control of short-term blood pressure]. Rev Neurol. 2014;59 (11):508-516. Spanish .

48. Heusser K, Tank J, Engeli S, et al. Carotid baroreceptor stimulation, sympathetic activity, baroreflex function, and blood pressure in hypertensive patients. Hypertension. 2010;55(3):619-626. doi:10.1161/HYPERTENSIONAHA.109.140665

49. Mannelli M, Pupilli C, Lanzillotti R, Ianni L, Serio M. Catecholamines and blood pressure regulation. Horm Res. 1990;34 (3-4):156-160. doi:10.1159/000181816

50. Ganten D, Stock G. Humoral and neurohormonal aspects of blood pressure regulation: focus on angiotensin. KlinWochenschr. 1978;56 (Suppl 1):31-41. doi:10.1007/BF01477450

\section{Publish your work in this journal}

Drug Design, Development and Therapy is an international, peerreviewed open-access journal that spans the spectrum of drug design and development through to clinical applications. Clinical outcomes, patient safety, and programs for the development and effective, safe, and sustained use of medicines are a feature of the journal, which has also been accepted for indexing on PubMed Central. The manuscript management system is completely online and includes a very quick and fair peer-review system, which is all easy to use. Visit http://www. dovepress.com/testimonials.php to read real quotes from published authors. 